\title{
"Iyambae": en busca de una educación superior emancipadora en la UNIBOL Guaraní y Pueblos de Tierras Bajas
}

\section{"Iyambae": in search of an emancipatory higher education in the UNIBOL Guarani and Lowland Peoples}

David Silvestre Delgadillo Zerda es profesor e investigador de la UIEP Universidad Intercultural del Estado de Puebla (México); candidato a doctor por la UNAM (México) (david.delgadillo@uiep.edu.mx) (http://orcid. org/0000-0002-5461-8450)

Recibido: 2017-08-12 / Revisado: 2017-11-17 / Aceptado: 2017-11-29 / Publicado: 2018-01-01

\section{Resumen}

En Latinoamérica (LA), hablar de las condiciones de posibilidad que den muestra de la construcción de una Educación propia en la educación superior como alternativa al proyecto pedagógico de la modernidad y del capitalismo hegemónico es referirse a experiencias educativas localizadas y concretas en la región. El contexto de diversidad corresponde al de las etnias indígenas de tierras bajas de Bolivia. Ahora bien, ¿Cuáles son los alcances y limitaciones que particularizan el abordaje de la diversidad étnica, cultural y lingüística del modelo de Universidad Indígena Boliviana (UNIBOL) Guaraní y de Pueblos de Tierras Bajas "Apiaguaki Tüpa" que permite hablar de la construcción de una educación propia orientada al posicionamiento de una pedagogía latinoamericana? Los pueblos indígena, originario, ancestrales (PIOAs) han impreso a esta institución las siguientes características: subversión de la configuración jerárquica herencia del modelo de universidad colonial (Weinberg, 1995), establecimiento de relaciones horizontales en un contexto de verticalidad de la educación superior (ES). Así como, la transformación de la función jerarquizante, monocultural y hegemonizante en una práctica pluralizadora y preservadora de la diversidad destinada a la superación de las desigualdades a través de su valoración rescate y fortalecimiento de elementos étnicos, culturales y lingüísticos propios. Además del tránsito de la suplantación de la presencia, voz y accionar de los PIOAs a un activo y efectivo protagonismo en la construcción de un modelo de ES constituido y constituyente de la visión histórica, política e identitaría de los pueblos indígenas de tierras bajas desempeñando una función emancipadora.

Descriptores: Educación propia, educación superior, pueblos indígenas, lenguas indígenas, emancipación, reivindicación política.

Forma sugerida de citar: Delgadillo, D. S. (2018). "Iyambae": en busca de una educación superior emancipadora en la UNIBOL Guaraní y Pueblos de Tierras Bajas. Alteridad, 13(1), 42-55. https://doi.org/10.17163/alt.v13n1.2018.03. 


\begin{abstract}
In Latin America (LA), to speak of the conditions of possibility that show the construction of an own education in higher education as an alternative to the pedagogical project of modernity and hegemonic capitalism is to refer to localized and concrete educational experiences in the region. The context of diversity corresponds to that of the indigenous lowland ethnics of Bolivia. Now, what are the scope and limitations that particularize the approach of the ethnic, cultural and linguistic diversity of the model of the Bolivian Indigenous University (UNIBOL) Guaran and of the Low Lands Peoples "Apiaguaki Tüpa" that allows to speak of the construction of an own education oriented to the positioning of a Latin American pedagogy? The indigenous, original, ancestral peoples (IOAPs) have printed to this institution the following characteristics:
\end{abstract}

\section{Introducción 1}

Actualmente, gran parte de la pedagogía que sustenta el accionar educativo en los países latinoamericanos adolece no solo de una fundamentación esencializada en el axioma de la desigualdad (Rancière, 2003). Modelo asumido y perfeccionado por el proyecto pedagógico de la modernidad y el capitalismo hegemónico (Laclau y Mouffe, 1985) sino también, instituye el sistema al cual representa, reproduce y disemina imponiéndola como el paradigma a emular en educación. Esquema que mantiene a los demás sistemas educativos en una situación de sometimiento, al modelo hegemónico, obligándolos a reproducir y perpetuar dicha desigualdad.

En educación quienes más han experimentado procesos de inferiorización y minorización, han sido los pueblos indígenas, concebidos, desde lógicas indigenistas (Castañeda, 2006) como incapaces para poder decidir sus destinos por sí mismos y más aún concebir, construir y llevar adelante sus propios sistemas educativos (Sarango, 2013). Proceder que ha encontrado en la educación superior, heredera de la colonia, el perfecto mecanismo de reproducción y naturalización de la relación de subalternización política, subversion of the hierarchical configuration, inheritance of the colonial university model; establishment of horizontal relationships in a context of verticality of higher education $(\mathrm{HE})$. As well as, the transformation of the hierarchical, monocultural and hegemonic function into a pluralizing and preserving practice of diversity destined to overcoming inequalities through its valuation, rescue and strengthening of own ethnic, cultural and linguistic elements. In addition to the transit of the supplanting of the presence, voice and actions of the IOAPs to an active and effective role in the construction of a model of HE constituted and constituent of the historical, political and identitarian vision of the indigenous peoples of the low lands performing an emancipatory function.

Keywords: Own education, higher education, indigenous peoples, indigenous languages, emancipation, political vindication.

económica, cultural, epistémica y lingüística de los pueblos indígenas.

En Bolivia la administración de Estado, arraigada en la colonialidad del poder (Quijano, y Wallerstein, 1992), negó históricamente el reconocimiento de dos elementos constituyentes e instituyentes de las naciones indígenas; sus territorios ancestrales y su libre determinación (Aparicio, 2009). Empero la lucha indígena, particularmente de tierras bajas, obtuvieron, en décadas recientes, logros importantes en la consecución de ambas reivindicaciones. Avance que configuró un espacio propicio para la concreción de las principales propuestas de la vía indígena erigiéndose como un proyecto social alterno.

La condición de mayoría es la que le permite a la sociedad indígena brindar su esquema interpretativo, su esquema de conocimiento y su posicionamiento político, como una posibilidad hegemónica que sea atractiva y que cuestione a los mestizos nuestra identidad. Para mí, lo más interesante políticamente del fenómeno de la insurgencia india, es que le plantea por primera vez al conjunto de la sociedad boliviana la posibilidad de indianizarse y de superar las visiones externas, esencialistas y cosificadoras de lo étnico (Rivera, 2008). 
En el ámbito de la educación superior la creación del régimen de universidades indígenas, particularmente la UNIBOL "Apiaguaki Tüpa" es la propuesta de los pueblos indígenas de tierras bajas. La trascendencia del proyecto educativo radica en la correlación entre el proyecto educativo y la visión política e ideológica propia de los pueblos indígenas de tierras bajas fundada en la reconstitución de los territorios ancestrales, la autodeterminación y la retoma de la historicidad propia. En este marco la experiencia desarrolla importantes elementos enmarcados en la construcción de una educación propia.

\section{Metodología}

Desde finales de los ochenta del siglo pasado, el accionar del movimiento indígena, además de otros factores confluyentes suscitaron cambios importantes en los países de la región; las respuestas oficiales del Estado-Nación involucraron procesos de institucionalización de la diversidad en la ES. Propuestas que van desde una lógica de inclusión, integración de población indígena (Mato, 2014, p. 43) hasta aquellas, procedentes de los mismos PIOAs consistentes en experiencias autogestivas. La diferencia entre ambas permite dilucidar el problema de investigación. Cuando el proyecto es planteado por no indígenas, entonces, el estudio se centraría en la pertinencia; pero, cuando la propuesta emerge de los mismos indígenas, el presente caso, el objetivo es evidenciar las posibilidades y limitaciones en el proceso de materializar y consolidar la proyección de la visión política, ideológica y cultural de estos pueblos en la ES.

La necesidad de conocer las particularidades que hacen de esta universidad una propuesta de los pueblos indígenas, instó adoptar la investigación cualitativa de corte etnográfico. La estrategia exploratoria implementó las siguientes técnicas: a) La revisión documental permitió acceder y analizar información relevante de la universidad. b) La entrevista fue de tipo no estructurada, en profundidad, y se aplicó bajo el criterio de mayor incidencia en la materiali- zación de la propuesta educativa. c) La observación no participante resultó vital para analizar los sucesos cotidianos; actitudes de detractores y/o partidarios del modelo. Los instrumentos utilizados fueron: el diario de campo, guías de entrevista; así como, el registro fotográfico y voz.

\section{Resultados}

\subsection{Contextualización de las naciona- lidades indígenas y las universi- dades interculturales en el Estado Plurinacional de Bolivia}

Bolivia es uno de los países más biodiversos del mundo, la profusión encarna también en la variedad étnica, cultural y lingüística. Una clasificación básica de la composición geográfica distribuye su territorio en "tierras altas" (puna, altiplano y valles) y "tierras bajas" (Amazonía, chaco y llanos orientales). Comparando ambas extensiones, el 60\% del territorio corresponde a la región de la Amazonía (Rivero, 2014), por tanto más que país andino debe ser considerado amazónico. La población total del país asciende a 10457329 habitantes, de los cuales el 44\% es indígena (INE, 2011), porcentaje cuestionado por la reducción drástica respecto del censo 2001 donde el $62 \%$ se auto-identificaba como indígena. Bolivia reconoce 36 nacionalidades indígenas, cinco habitan ancestralmente en tierras altas: El resto, 31 están localizados en tierras bajas. Esto indica una mayor concentración de la diversidad étnica, cultural y lingüística en tierras bajas

La instrumentalización de la educación originada en la colonia y proseguida en la época republicana busco siempre la desestructuración étnica, política, territorial y simbólica de PIOAs imponiéndoles una política educativa homogeneizadora, minorizadora e inferiorizante (Choque, 2015) denominada Indigenismo (Fabre, 1998). A contracorriente, la subalternización provocó en los indígenas la necesidad de apropiarse de la educación. Para el movimiento indígena la paradoja expuso connotaciones subversivas sobre la educación asumiéndosela cómo 
una más de sus demandas (Rojas, 2003). En este sentido, la lucha de los pueblos indígenas por sus reivindicaciones generó en las últimas cuatro décadas cambios importantes. Transformaciones que alcanzaron su punto más alto con la aprobación de una Nueva Constitución Política del Estado (NCPE) en 2009. Acuerdo social donde la participación activa de los pueblos indígenas fijó las bases del nuevo modelo de Estado fundadas en el reconocimiento de la plurinacionalidad que lo obliga a atender las demandas, necesidades y expectativas de los pueblos que la conforman.

Con la creación del régimen de universidades indígenas bolivianas en 2008 el escenario de la educación superior experimenta cambios significativos que impulsa la explicitación de la obligatoriedad del Estado a garantizar a las nacionalidades indígenas el rescate, mantenimiento y desarrollo de la diversidad étnica, cultural y lingüística queda establecida en la NCPE:

La educación superior es intracultural, intercultural y plurilingüe, y tiene por misión la formación integral de recursos humanos con alta calificación y competencia profesional; desarrollar procesos de investigación científica para resolver problemas de la base productiva y de su entorno social; promover políticas de extensión e interacción social para fortalecer la diversidad científica, cultural y lingüística; participar junto a su pueblo en todos los procesos de liberación social, para construir una sociedad con mayor equidad y justicia social (Gaceta oficial del Estado Plurinacional de Bolivia, 2009, p. 25).

Asimismo, desde 2011 entra en vigencia, la Ley de Educación "Avelino Siñani, Elizardo Pérez" incorporando nuevos elementos tendientes a preservar y fortalecer el patrimonio cultural de los PIOAS. El artículo $5^{\circ}$ fija principios que regirán la educación acorde al nuevo modelo de país establecido por la constitución señalando; "Las naciones y pueblos indígena originario campesinos tienen derecho a una educación intracultural, intercultural y plurilingüe en todo el sistema educativo." Con esto se busca que todo el sistema educativo contribuya al fortalecimiento de la unidad e identidad de los pueblos indígenas como parte del Estado Plurinacional, así como a la identidad y desarrollo cultural de sus miembros.

UNIBOL Guaraní y de Pueblos de Tierras Bajas "Apiaguaiki Tüpa"

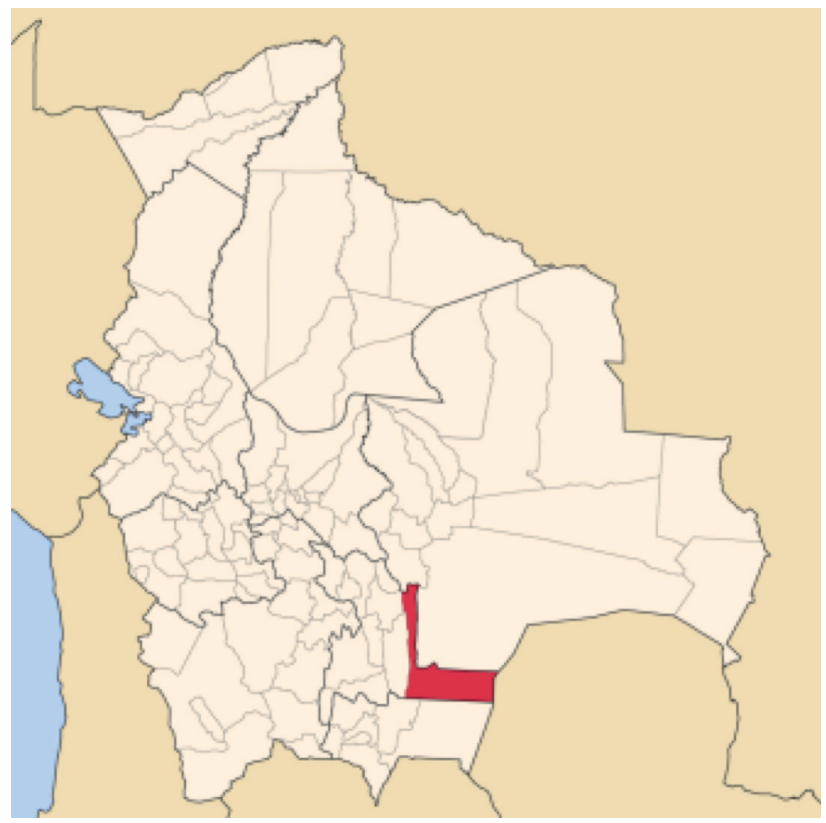


Enclavada en la comunidad de Ivo, provincia Luis Calvo del Departamento de Chuquisaca, en el territorio indígena originario (TIO) Guaraní, se encuentra el campus de la UNIBOL "Apiaguaiki Tüpa”. Desde su fundación esta universidad mostró avances importantes, remitiéndonos a la posibilidad de construcción de una educación insumisa (Medina, 2015) y con ella una pedagogía alterna. En este sentido, la visualización del proyecto político de los pueblos indígenas está esencializada en dos condiciones básicas de vida para la población indígena: el territorio y la emancipación. El carácter vital de estos elementos se tradujo para los pueblos indígenas en dos imperativos; el primero, "la reconstitución de los territorios indígenas" y el segundo "la autodeterminación de estos pueblos". Ambos preceptos enmarcados en la retoma de la historicidad propia (Cusicanqui, 2010, p. 54).

Bajo estos dos principios ordenadores la población indígena generó numerosas y diversas estrategias de resistencia y lucha para enfrentar las condiciones brutales de desestructuración sociocomunitaria cultural impuestas por el sistema colonial (Golte, 1980) que significó la usurpación y despojo de los territorios indígenas ancestrales y la explotación de sus recursos naturales. Además del sometimiento de la población indígena a trabajos forzados bajo modalidades de servidumbre y esclavitud que duró casi tres siglos, desde la llegada de los españoles hasta 1825 (Canedo, 2011). La independencia, reafirmando su herencia colonial, no puso fin al despojo y la explotación, intensificándola. Situación que afirmo la reconstitución territorial y la autodeterminación como reivindicaciones inequívocas de los PIOAs. Para los indígenas de tierras bajas de Bolivia, los movimientos milenaristas (Wachtel, 1973) como el Ivi Marae "tierra sin mal" para los guaraníes; o el Kandire "La Búsqueda de la Loma Santa" para los moxeños constituyen la estrategia relacionada con la reconstitución de los territorios indígenas (Lehm, 1991).

Los territorios son espacios sagrados donde las personas o las familias guaraníes tienen que cuidar y formar parte de ella... entonces esas tres categorías es, para nosotros, es que necesariamente tiene que tener un territorio ¿no? y si no cumple esos tres componentes el territorio está en conflicto, si solo es un pequeño lotecito para la casa, le falta algo ¿no?, le falta tener un sembradío. Pero si tengo casa y luego un sembradío, resulta que me está faltando algo que es más importante ¿no? que es el espacio mayor que es el lugar sagrado, lugar para la caza, lugar para la espiritualidad, para el desplazamiento de las personas y el contacto también con otros seres, entonces ese es el espacio mayor, entonces en esas tres dimensiones se entiende el territorio y bueno desde tiempos muy antiguos hemos estado siempre buscando territorio así ¿no? la palabra buscar, la búsqueda del Ivi Marae o "Tierra sin Mal” no significa que no lo teníamos ¿no? sino que era un permanente estar en ese espacio de la tierra sin mal. En otro momento si requería, cuando ya el territorio era desgastado, el territorio ya no tenía esos tres elementos era necesario un desplazamiento o el uso espacial dentro el dominio espacial sobre sus territorios. Entonces esa es la visión de la tierra sin mal ¿no? para nosotros (E. Camargo, entrevista, 18/11/2014).

En el mismo sentido el Iyambae o Iyaambae cuyo significado más cercano refiere a un ser humano libre "el sin dueño" se establece como estrategia que insta a la búsqueda de autodeterminación de los pueblos indígenas. Ambos preceptos impulsan y orientan históricamente el accionar y vida de la población indígena hacia la consecución de estos ideales.

Iyambae es una condición, es una condición humana, no solo del guaraní sino lo que decimos es que el guaraní es libre, sin dueño, autónomo, libre para decidir su futuro libremente. Ese es el ser Iyambae no tendría que haber patrón, no tendría que haber alguien que dirija. Entonces esa es también la dignidad de las personas ¿no? Porque el mundo ahora ha entrado hay unos que explotan y otros que...un explotador y otros que son explotados. Entonces en la visión guaraní no es eso ¿no? sino el ser soberano en sí, la persona. Por eso es que antes no teníamos... una confede- 
ración, de los guaraníes antes, no había un... alguien que mande a toda la nación guaraní, sino que cada sector, cada comunidad, cada persona, cada capitanía era libre ¿no? claro en el momento de defender el territorio se unían toditos pero después cada quien a desarrollar de manera autónoma su propia vida ¿no? entonces esa es una de las cuestiones más importantes que nosotros cuidamos ¿no? (E. Camargo, entrevista personal, 18/11/2014).

Impulsados y direccionados por estas utopías movilizadoras los PIOAs han obtenido, en las tres últimas décadas, importantes resultados en la consecución de sus reivindicaciones fundamentales. Entre los logros perceptibles para los pueblos indígenas encontramos: 1) Obtienen la titulación colectiva de 20715 950,3 hectáreas desde 1990 hasta 2010 en calidad de Territorios Indígena Originario Campesinos (TICOs), Fundación Tierra (2011). 2) La elección del primer presidente indígena en 2006. 3) En 1985 Proponen se convoque a la asamblea constituyente. 4) Participan junto a otras organizaciones indígenas y de la sociedad civil en la realización de Asamblea Constituyente de 2006 a 2009. 5) Logran el reconocimiento de la composición étnica, cultural y lingüística diversa del país cambiando la denominación de República de Bolivia por el de Estado Plurinacional de Bolivia (EPB). 6) Consiguen la constitucionalización de sus demandas históricas como el reconocimiento del "territorio indígena originario campesino” y la autonomía indígena. 7) Obtienen la representación política directa en el órgano legislativo. 8) Constitucionalizan la consulta previa en la carta magna.

Dada la existencia precolonial de las naciones y pueblos indígena originario campesinos $\mathrm{y}$ su dominio ancestral sobre sus territorios, se garantiza su libre determinación en el marco de la unidad del Estado, que consiste en su derecho a la autonomía, al autogobierno, a su cultura, al reconocimiento de sus instituciones y a la consolidación de sus entidades territoriales, conforme a esta Constitución y la ley (Gaceta del Estado Plurinacional de Bolivia, 2009.17).
Así el movimiento indígena habría avanzado, tanto en la reconstitución de los territorios ancestrales como en la autodeterminación de los pueblos. En este sentido los logros han redundado en el reconocimiento y revalorización de la diversidad étnica, cultural y lingüística de las nacionalidades indígenas por el conjunto de la sociedad boliviana en sus diferentes estamentos sociales. Hecho que permitió acordar un nuevo pacto social bajo la consigna "nunca más sin los pueblos indígenas." De este modo los pueblos indígenas han incrementado su visibilización y participación en diferentes ámbitos político, jurídico, económico y social en Bolivia. Asimismo, la elección del primer presidente indígena de Bolivia en 2006 marca un continuo en las concreciones de varias demandas históricas sujetas siempre a las utopías movilizadoras (Ivi marae e Iyambae). Configuración propicia para la instauración de las principales propuestas de la vía indígena en diferentes esferas sociales.

En el ámbito de la educación superior en 2008 se crean tres universidades indígenas: la UNIBOL aimara “Tupak Katari”, la UNIBOL quechua "Casimiro Huanca" y la UNIBOL Guaraní y de Pueblos de Tierras Bajas "Apiaguaki Tüpa". La creación de estas IES se inscribe en la visión política amplia de los pueblos indígenas orientados siempre a la reconstitución ancestral de los territorios ancestrales, la autodeterminación de las naciones indígenas y la retoma de la historicidad de los pueblos indígenas. La creación de este nuevo régimen de universidades ${ }^{2}$ alternas a las universidades clásicas, convencionales ya existentes se justifica en la experiencia de gran parte de la población indígena que tuvo que lidiar con procesos de alienación, pérdida de identidad cultural, priorización de intereses individuales y una visión predominantemente comercial del conocimiento, bajo un principio monocultural (Díaz-Polanco, 2006) que invisibilizó y descalifico el conocimiento y comprensión de la realidad producido por los pueblos indígenas.

La crítica al régimen de universidades públicas-convencionales, atrincherada en la 
autonomía universitaria, deviene no solo de su invariable función reproductora de la desigualdad arraigada fuertemente en el pensamiento colonial (Tünnermann, 2010). Sino también del inexistente aporte en los procesos de transformación emprendidos por los sectores sociales mayoritarios desde 2004 que culminaron con la aprobación de la NCPE en 2009, más aún prevalece la actitud reticente a los cambios propugnados por sectores sociales mayoritario. Negativa que repercutió en la necesidad de crear IES (no convencionales) indígenas correlacionadas a los requerimientos educativos del ser, actuar y pensar propio de la alteridad indígena.

Con la puesta en marcha de la UNIBOL "Apiaguaiki Tüpa" se expone la tensión existente entre dos modelos de universidad que pugnan por imponerse al interior de la institución. Por un lado está el modelo de universidad convencional moderna de corte occidental, con el que vienen funcionando las universidades públicas y privadas, desde la fundación de la primera universidad en Bolivia. Por otro lado está el "modelo de universidad indígena no convencional" que podría describirse como el intento de concreción de la idea de educación superior propia que tienen los pueblos indígenas, en este caso los pueblos indígenas de tierras bajas de Bolivia.

Al no haber un modelo preestablecido de universidad indígena, sino que está en construcción, la consolidación en la experiencia de la UNIBOL se enfrenta a la influencia que ejerce el modelo convencional preestablecido. En este sentido la convergencia de ambos modelos al interior de esta IES indígena expresa sus múltiples tensiones y conflictos en distintas dimensiones, niveles y actores universitarios impactando en el desarrollo constitutivo de la educación propia en el nivel superior de la educación consignada bajo esta IES. Tal coyuntura configura los alcances y límites de dicha experiencia afectando diferentes componentes constitutivos, estos son: a) Participación de los pueblos indígenas en la estructura de gobierno universitario y funcionamiento actual de la universidad, b) El diálogo y articulación del conocimiento universal de corte occidental con los saberes y conocimientos propios, c) Dinámica sociolingüística y abordaje institucional de las lenguas indígenas; y d) la vinculación universitaria con las comunidades, organizaciones y población indígena.

\subsection{Participación de los pueblos indí- genas en la estructura de gobierno y funcionamiento actual de la uni- versidad de la UNIBOL Guaraní y de pueblos de Tierras Bajas}

Uno de los rasgos más visibles de la UNIBOL Guaraní desde su creación hasta el momento, es la implicación de la población indígena en el proyecto. Actualmente alberga a 732 estudiantes y 62 docentes. La evidencia constata una participación cualitativa en cuanto plantea y materializa una propuesta académica posicionada en la visión política e ideológica de los pueblos de tierras bajas. Cuantitativamente la participación de los PIOAs se efectiviza, en cuanto el planteamiento educativo es vehiculizado en instancias de proposición, decisión y ejecución donde la participación de los pueblos indígenas es mayoritaria definiendo el curso de acción institucional. La propuesta educativa ciertamente está a cargo de los pueblos indígenas de tierras bajas; tanto en la estructura de gobierno como la administración de recursos, la gestión institucional y académica. Los mismos actores indígenas visualizan a la universidad como una institucionalidad creada por ellos. Igualmente, la población no indígena reconoce a esta IES como una institucionalidad propiamente indígena.

La máxima instancia de decisión en la UNIBOL “Apiaguaki Tüpa”, en la estructura organizacional, está dada por la Junta Comunitaria. Esta instancia está conformada por los representantes de cada uno de los pueblos indígenas de donde provienen los estudiantes. Si hay 12 nacionalidades indígenas presentes en la universidad, serán 12 representantes indígenas en el consejo, los directores de cada las cuatro 
carreras; Ingeniería en Petróleo y Gas Natural IPGN, Ingeniería Forestal (IF), Ingeniería en Eco-piscicultura (IEP) y Medicina Veterinaria (MV). Un representante docente por carrera (4 docentes), un representante estudiante también por carreras (4 estudiantes indígenas) y el Rector o Rectora, indígena. Esta composición señala que la tuición de la universidad recae mayoritariamente en la población indígena haciendo de la participación indígena deliberativa y decisoria.

\subsubsection{La UNIBOL Guaraní de Tierras Bajas está} en directa vinculación con las demandas mediatas, inmediatas y posteriores de los pueblos indígenas del oriente boliviano

Bajo el lema "por el territorio y la dignidad" en 1990 en Bolivia, el movimiento indígena de tierras bajas convocó a una marcha reivindicando el derecho de los pueblos indígenas al territorio. Con esta marcha los pueblos originarios lograron que el gobierno les titulara en calidad de TCO (Tierras Comunitarias de Origen) tres extensiones territoriales (Lehm, 1991). Desde entonces se han reconocido en Bolivia más veinte TCOs a diferentes nacionalidades indígenas.

En esta coyuntura la creación de las universidades indígenas constituye una respuesta a las necesidades educativas de los PIOAs vinculadas esencialmente a la ocupación y aprovechamiento de recursos naturales en sus territorios ancestrales. En este sentido, la creación de los cuatro programas educativos convencionales responde a la exigencia constitucional de impulsar la productividad a través de la ES. Igualmente, corresponden con los recursos naturales existentes en los territorios: Ingeniería del Petróleo y Gas Natural, vinculadas al aprovechamiento de los hidrocarburos cuyos yacimientos importantes se encuentran ubicados en los territorios indígenas de la nación Guaraní. Ingeniería Forestal, relacionada al aprovechamiento de los recursos forestales existentes en los bosques de la Amazonía. Ingeniería en Ecopiscicultura relacionada con el aprovechamiento de los recursos hídricos abundantes en el oriente boliviano. Y Medicina Veterinaria previendo la potencialidad de cría de animales.

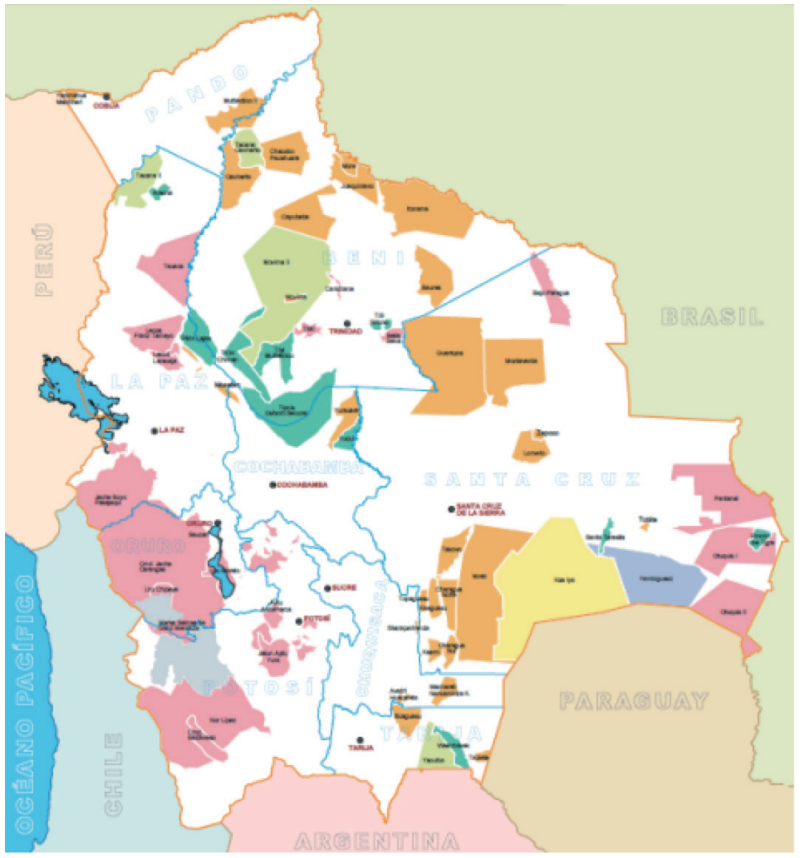




\subsubsection{Los sabios indígenas en la UNIBOL Guarani y de Tierras Bajas}

Un rasgo a destacar de la experiencia educativa es la emergencia, en el ámbito académico, de los sabios indígenas, actualmente en la universidad están trabajando bajo esta función cuatro sabios que corresponden a las naciones indígenas: guaraní, moxeño, bésiro y guarayu. En cuanto a sus funciones son varias; orientador para los estudiantes, educador, experto en lengua indígena, desempeñándose también como asesores académicos y políticos que guían a los órganos de gobierno y de administración institucional. De entre todas las funciones realizadas por los sabios indígenas sobresalen dos: la primera relacionada con el rol de depositarios de los saberes y conocimientos de los pueblos indígenas y mediadores en los procesos de articulación entre el conocimiento universal de corte occidental y los saberes y conocimientos propios. En este sentido la importancia de la función pedagógica del sabio indígena para la UNIBOL radica en su potencialidad como agente intraculturizador en procesos académicos de diseño y restructuración curricular de planes y programas académicos. Intervención indispensable en la tarea de promover un pluralismo epistemológico (Prada, 2015) en un contexto adverso, por tratarse de carreras convencionales cuyos campos de conocimiento están conformados por la ciencia estructural, los métodos explicativos basados en la totalidad, y el método científico de corte occidental como medio de validación.

La segunda función está relacionada con su papel de guía y consejero político en la conducción de la UNIBOL, allí se constituye en vigilante de la junta comunitaria. Precautelando que los procesos académicas, administrativos, sociales, culturales no contravengan a la propuesta educativa indígena establecida por la institución. En este sentido aboga en diferentes instancias, especialmente en los diferendos académicos entre docentes técnicos (anclados en formatos académicos convencionales), docentes de len- guas indígenas (posicionados en la propuesta indígena). $Y$ docentes transversales (custodios de la propuesta) cuidando que la consolidación del modelo mantenga las características que la hacen indígena.

\subsubsection{Dinámica sociolingüística y abordaje ins- titucional de las lenguas indígenas en la UNIBOL "Apiaguaiki Tüpa"}

De las tres universidades indígenas creadas en Bolivia la UNIBOL "Apiaguaiki Tüpa" es la que más diversidad étnica, cultural, lingüística atiende. Esto le significa a la institución una gran dificultad y un verdadero desafío referido al rescate y fortalecimiento de las lenguas indígenas de tierras bajas. Actualmente en la Universidad podemos encontrar estudiantes provenientes de 17 naciones indígenas, incluidos algunos estudiantes provenientes de tierras altas, de lengua aimara y quechua.

No obstante, la complejidad que involucra revitalizar el uso de las lenguas indígenas la UNIBOL ha asumido este reto, estableciendo la asignatura de lengua originaria (indígena) como trocal en las cuatro carreras; con el objetivo de producir investigaciones, ciencia y teoría desde la lengua indígena. Así los estudiantes puedan elaborar y teorizar desde su propio código lingüístico, además de construir representaciones del contexto productivo, ambiental, tomando en cuenta su propia forma de ver el mundo vehiculizado por su lengua como instrumento principal (UNIBOL, 2012).

El abordaje de la lengua indígena en la institución muestra dificultades y limitaciones referidas principalmente a la imposibilidad de atender adecuadamente la gran diversidad de lenguas presentes en la UNIBOL. Si bien la intención es consolidar un modelo de revitalización de las lenguas de tierras bajas, materialmente esto no es posible porque implica la contratación de por lo menos una treintena de docentes sólo para este eje curricular. Por esta razón la institución optó por atender gradualmente a las lenguas en la formación profesional. Inicialmente trabaja 
cuatro lenguas indígenas de uso mayoritario en los estudiantes: el guaraní, el moxeño, el bésiro y el guarayu. Las demás lenguas se irán abordando en los subsiguientes años de forma gradual.

Numéricamente los estudiantes bilingües (Appel y Muysken, 1997) son pocos en comparación con los estudiantes monolingües en castellano en la UNIBOL. La estrategia adoptada consiste en agrupar a los estudiantes en bilingües castellano-lengua indígena y en monolingües en castellano. Los primeros llegan a la universidad con todas las habilidades lingüísticas en castellano y ciertas competencias en lengua indígena. En cambios los segundos vienen solo con el dominio de las competencias en castellano (entienden, hablan, escriben y leen). Ambos grupos abordan la lengua con diferentes finalidades los dos primeros semestres; los bilingües buscan consolidar las cuatro habilidades lingüísticas y los monolingües inician el aprendizaje de la lengua, esto supone inicialmente la elección de una de las cuatro lenguas ofertadas por la institución. En tercer semestre, se fusionan ambos y conforman grupos de cooperación para la consolidación de las competencias en lengua indígena, ahí los estudiantes bilingües asumen un rol importante como apoyo en la formación de sus compañeros monolingües. Pese al tiempo dedicado y la estrategia adoptada para favorecer el aprendizaje de las lenguas, los resultados aún son muy limitados, al terminar su profesionalización los estudiantes (monolingües) aún no han logrado consolidar las capacidades lingüística en lengua indígena.

\subsubsection{Tendencia de los docentes a replicar la función docente convencional de su propia formación profesional}

Todos los docentes de los cuatro programas de la UNIBOL "Apiaguaiki Tüpa” se formaron en universidades públicas convencionales, su práctica docente actual refleja mucho de su experiencia de formación profesional caracterizado por la función de maestro explicador reproductora de la desigualdad de inteligencias (Ranciére,
2003). Donde el rol activo estaba centrado en la enseñanza del docente, sus conocimientos, su método, etc. el modelo de universidad indígena proporciona a estos docentes el espacio para transformar su experiencia docente subordinadora de inteligencias a una práctica educativa emancipadora.

Al momento la UNIBOL presenta tres tipos de docencia de acuerdo a las áreas curriculares: docentes técnicos, docentes de lenguas indígenas y docentes transversales. Debido a la formación de los docentes en universidades convencionales, el modelo explicador estuvo presente en todos pero con diferenciadas intensidades. Así los docentes de las ingenierías parecen haber experimentado, acentuadamente, procesos de atontamiento pedagógico (Ranciére, 2003). La razón remite a los contenidos del área de las ciencias naturales fuertemente estructurados en los métodos de comprobación científica. De ahí la reticencia a concebir otros sistemas de conocimientos cómo válidos, principio transformador de la práctica educativa. En cambio los docentes el área transversal parecen haber sido menos afectados por la experiencia educativa del maestro explicador, debido en parte a la naturaleza de los contenidos que corresponden con el área de las humanidades y las ciencias sociales. Su práctica actual como docentes muestra una mayor apertura a la emancipación de la educación, en muchos casos, promueve abiertamente e instan a los docentes del área técnica a transitar de una metodología de enseñanza fundada en la desigualdad de inteligencias a una metodología de la igualdad de voluntades. Por su parte los docentes de lengua indígena, fortalecidos en su identidad cultural se constituyen en promotores del modelo indígena.

Las negociaciones entre estos tipos de docentes perfilan una determinada articulación de saberes, conocimientos y lenguas producto no siempre del diálogo sino como resultado de la pugna por asignar un porcentaje de la calificación distributiva que refleje no solo el dominio de conocimientos del área disciplinar técnica, 
sino también, el uso académico de la lengua indígena, la fundamentación cultural que recupera la propia historicidad, además de la recuperación de algún saber o conocimiento propio.

\subsubsection{Los contenidos de algunas asignaturas se prestan más al método explicador}

En cuanto a los contenidos de las diferentes asignaturas de la carrera de ingeniería forestal de la UNIBOL cabe señalar la presencia de contenidos en áreas curriculares que justificarían prácticas docentes tradicionales. Dichos contenidos corresponden al área de las ciencias naturales. Disciplinas como dendrología, edafología, botánica general, fisiología vegetal y otras propias del área forestal, así como las materias de física, química y cálculo cuyos contenidos están cimentados en métodos rígidos de comprobación y clasificación científica. Por tanto, el peso de estas asignaturas en la formación profesional viene revestido por un estatus de ciencia indiscutible frente a otros saberes y conocimientos en particular, los indígenas. En este sentido los docentes técnicos muchas veces descalifican los conocimientos provenientes del patrimonio cultural de los estudiantes negándose a considerarlos como conocimientos, especialmente en trabajos de investigación de grado. Asimismo la concepción de superioridad de los conocimientos "científicos" de estas las asignaturas es transmitida a los estudiantes, en este sentido se replica la jerarquización "desigualdad del conocimiento". Dicho sea de paso, en estas asignaturas el porcentaje de aprobación del curso por tradición, es menor al de otras.

Por otro lado, en los estudiantes se puede observar también, el peso de una trayectoria educativa, en los niveles escolarizados, caracterizada por la práctica pedagógica explicadora que ha trastocado el modo de concebir el proceso de aprendizaje. Las repercusiones de esta experiencia se pueden percibir en la inseguridad que los estudiantes muestran a la hora de enfrentarse a tareas académicas donde la intervención del docente se reduce. Al respecto López (2009) señala que el peor empobrecimiento es que hemos formado a la población indígena o de ancestro indígena como gente insegura de sí misma, y un sujeto inseguro no puede contribuir al desarrollo de un país. Asimismo, otra implicación de la experiencia educativa escolarizada, es que para gran parte de los estudiantes su aprendizaje deviene de la explicación de un docente quien tiene consolidado un gran cúmulo de conocimientos en el área que enseña. Especialmente cuando se trata de las materias del área técnica.

Cuando se les pregunta ¿cuáles son las características de los maestros que enseñan bien? Muchos responden que son aquellos quiénes explican mejor, adjudicando su aprendizaje no a sí mismos sino al rol explicador del maestro; así como a su cúmulo de conocimientos del área y que están en permanente actualización. La concepción misma del aprendizaje de los estudiantes alude a ese proceso de vaciamiento del conocimiento del docente hacia los estudiantes, elementos que refuerzan y mantienen el rol explicador del maestro.

\section{Discusión}

No obstante los cambios del Estado logrados por los movimientos sociales, especialmente, el movimiento indígena. La transformación de la educación superior en Bolivia ha encontrado en el modelo de universidad convencional, tradicional, autónoma, de origen colonial un obstáculo infranqueable para concretar la visión educativa propia. De ahí que la reconfiguración de la educación superior haya buscado otros derroteros, como la creación de otros regímenes de universidades entre las que se encuentran las universidades indígenas. La institucionalidad de las IES Indígenas trasciende la tradicional autonomía universitaria, enmarcándose en una autonomía más amplia la "autonomía indígena".

En el proyecto de educación superior indígena no hay interpretación de los requerimientos educativos de los pueblos indígenas de tierras bajas. Tampoco existe una intermediación agen- 
tiva, pues son los mismos indígenas los ideólogos, propulsores e interlocutores quienes, a través de sus organizaciones, transmiten, negocian con el Estado sus demandas, necesidades y expectativas educativas. Actuar que les ha permitido llevar adelante sus propias propuestas de educación superior. En este sentido, la participación de la población indígena fue decisiva desde el inicio, en la gestación la idea y creación de la UNIBOL "Apiaguaiki Tüpa" y su actual desarrollo.

Los actores indígenas universitarios tienen claro y asumen la diferencia con las universidades públicas convencionales, las cuales constituyen el referente inicial para la construcción de una propia identidad institucional. Sin embargo, se observa una resistencia al cambio por parte de algunos docentes, quienes, debido a su formación en IES convencionales, no asumen aun el enfoque indígena propugnado por la institución y las organizaciones indígenas desarrollando sus actividades académicas en un marco aun tradicionalconvencional de educación superior. Ello dificulta la consolidación de un modelo propio en la ES.

La elección explícita de la propuesta educativa superior por la vía indígena permite a los actores involucrados, desde el inicio, Identificar las demandas, necesidades y expectativas que los PIOAs confieren a la educación superior. Hecho que faculta a los participantes posicionarse críticamente, identificándose con la visión política ideológica de los pueblos indígenas a la que responde la propuesta educativa de esta universidad. Asimismo, ello repercute en el involucramiento y compromiso de los actores universitarios en el proyecto educativo.

La atención exclusiva de la población indígena en la ES, a través del modelo indígena, reditúa en la claridad de los involucrados, organizaciones indígenas, docentes, comunidad, estudiantes, autoridades de su accionar político, pedagógico y epistemológico porque los fines, objetivos y propósitos están directamente vinculados a la consecución de las reivindicaciones históricas de los pueblos indígenas y la proyección de los horizontes ontológicos semióticos
(De Alba, 2009) de los 31 pueblos indígenas de tierras bajas.

\section{Conclusiones}

Desde la época colonial, la educación indígena en Bolivia ha sido negada y perseguida; no es sino, en las últimas cuatro décadas que la lucha del movimiento indígena concretó avances importantes en varios ámbitos sociales. La consolidación de la propuesta de educación superior indígena, evidencia de este avance en el ámbito educativo, muestra elementos significativos en cuanto los rasgos que la particularizan corresponden al locus de enunciación de los PIOAs, identificar estos elementos y su lógica de articulación. Permite conocer el curso en la construcción de una educación propia cuya teorización aporte a la emancipación pedagógica Latinoamericana.

Como se observa, la emergencia de la UNIBOL Guaraní de Pueblos de Tierras Bajas se opone a la función suplantadora anclada en la desigualdad, y apuesta más bien por una práctica pedagógica sustentada en la valoración de los saberes y conocimientos de los pueblos indígenas. Este posicionamiento implica un cambio que orienta a la institución en su accionar pedagógico hacia la búsqueda del establecimiento de vínculos con las comunidades y la población indígena de las diferentes nacionalidades indígenas de tierras bajas valorándolas como poseedoras de un legado cultural traducido en saberes, conocimientos y tecnologías ancestrales. Patrimonio cultural que corresponde a cada uno de los pueblos indígenas de los cuales provienen los estudiantes de esta universidad, convirtiéndolos en sus portadores. Por lo tanto los protagonistas de los procesos de formación profesional (autoridades, docentes y alumnos) deben aspirar a establecer una metodología de aprendizaje que suscite la emancipación política, intelectual, cultural y lingüística de los estudiantes.

Cabe resaltar que este fin pedagógico constituye una parte importante del proyecto educativo de los pueblos indígenas, quienes lle- 
van construyendo en Bolivia y otros países de Latinoamérica proyectos referidos a la educación propia: la de los pueblos indígenas-originarios. Asimismo, como se puede observar la educación propia no implica una esencialización de las culturas indígenas ancestrales que represente un retorno al pasado. Se trata más bien de resignificar la presencia viva de los pueblos indígenas en el mundo actual dotándola de sentido propio mediante la retoma de su historicidad. Ello implica, una necesaria interrelación con otras visiones de mundo, incluso hegemónicas que están modelando las formas actuales de vida, buscando establecer una interculturalidad crítica (Walsh, 2002) que les permita emanciparse del dominio cultural, ideológico, político y lingüístico impuesto históricamente.

La elección explícita de la propuesta educativa superior por la vía indígena permite a los actores involucrados, identificar claramente las demandas, necesidades y expectativas que los pueblos indígenas le confieren a la educación superior en el marco de su historicidad. Hecho que faculta a los participantes posicionarse críticamente, posicionándose desde la visión política ideológica a la que responde la propuesta educativa de esta universidad. Ello repercute en el involucramiento y compromiso de los actores universitarios en el proyecto educativo.

\section{Notas}

1 Proyecto subvencionado por el Consejo Nacional de Ciencia y Tecnología (CONACYT) de México, dentro del proyecto “Construcción de la educación propia en las IES: un estudio comparativo de dos universidades: La Universidad Indígena Boliviana Guaraní y de Pueblos de Tierras Bajas “Apiaguaiki Tüpa” (Bolivia) y La Universidad Veracruzana Intercultural (México)".

2 Artículo $1^{\circ}$ del decreto supremo № 29664 de creación de las tres universidades indígenas bolivianas de fecha 2 de agosto de 2008 “Créase tres (3) Universidades Indígenas Bolivianas Comunitarias Interculturales Productivas-UNIBOL "Aymara", "Quechua" y "Guaraní y Pueblos de Tierras Bajas" como entidades descentralizadas de educación pública superior, bajo Régimen Especial y tuición del Ministerio de Educación y Culturas. Gaceta oficial del Estado Plurinacional de Bolivia.

\section{Bibliografía}

Aparicio, M. (2009). La libre determinación y la autonomía de los pueblos indígenas. El caso de México. Boletín Méxicano de Derecho Comparado, 42(124), 13-38. Recuperado de https://goo.gl/zMzuJ1

Appel R. y Muysken P. (1997). Bilingüismo y contacto de lenguas. Córcega- Barcelona: Ariel.

Canedo, G. (2011). La loma santa: una utopía cercada. Territorio, cultura y estado en la amazonía boliviana. La Paz: IBIS-Plural.

Castañeda, E. (2006). La educación indígena en México. Ciudad de México: Universidad Nacional Autónoma de México.

Choque, María E. (2015). La estructura universitaria vigente, resabio del colonialismo, te contradice y te anula como ser humano. En: Daniel Mato (Coord.), Educación Superior y Pueblos Indígenas en América Latina. Buenos Aires: EDUNTREF.

De Alba, A. (2009). Horizonte ontológico semiótico en la relación espacio epistémico-teoría. Recuperado de https://goo.gl/gCJzKR

Díaz-Polanco, H. (2006). Elogio a la diversidad. Globalización, multiculturalismo y etnofagía. México: Siglo XXI Editores.

Gaceta Oficial del Estado Plurinacional de Bolivia (2008). Decreto 29664 de creación de tres universidades indígenas bolivianas. La Paz-Bolivia.

Gaceta Oficial del Estado Plurinacional de Bolivia (2009). Constitución Política del Estado Plurinacional de Bolivia. La Paz-Bolivia.

Golte, J. (1980). Protesta contra los repartimientos mercantiles en sus repartos y rebeliones. Tupac Amaru y las contradicciones de la economía colonial. Lima: IEP.

Laclau, E. y Mouffe, Ch. (1985). Hegemony and Socialist Strategy: Towards a Radical Democratic Politics. London: Verso.

López, LE. (2009). Pueblos indígenas y educación superior en América Latina y El Caribe. En: Interculturalidad, educación y ciudadanía. La Paz-Bolivia: Plural Editores.

Lehm, Z. (1991). Loma santa: procesos de reducción, dispersión y reocupación del espacio de los pueblos indígenas mojeños. Tesis. La PazBolivia: UMSA. 
Ministerio de Educación (2010). Ley de la educación "Avelino Siñani- Elizardo Pérez" Nº70.

Mato, D. (2014). Universidades Indígenas en América Latina. Experiencias, logros, problemas, conflictos y desafíos. ISEES Inclusión Social y Equidad en la Educación Superior, (14), 17-45. Recuperado de https://goo.gl/4raZ3o

Medina, P. (2015) Pedagogías insumisas: movimientos político-pedagógicos y memorias colectivas de educaciones otras en América Latina. México: Universidad de Ciencias y Artes de Chiapas.

Ministerio de Educación (2012). Plan de Estudios de la Carrera de Ingeniería Forestal Universidad Indígena Boliviana (UNIBOL) Guaraní y Pueblos de Tierras Bajas "Apiaguaki Tüpa". Chuquisaca-Bolivia.

Prada, R. (2015). Epistemología, pluralismo y descolonización. Cochabamba: FUNPROEIB Andes.

Quijano, A. y Wallerstein, I. (1992). Americanity as a concept or the Americas in the modern world-system. International Social Science Journal, (134), 549-557.

Rancière, J. (2003). El maestro ignorante. Cinco lecciones sobre la emancipación intelectual. Barcelona: Editorial Laertes.

Rojas, A. (2003). La etnoeducación y construcción de sentidos sociales. Memorias. Popayan: Instituto Caro y Cuervo, CCELA, PROEIB Andes.

Rivera, S. (2008).Violencia e interculturalidad. Paradojas de la etnicidad en la Bolivia de hoy. Recuperado de https://goo.gl/EhsLXK

Sarango, L.F. (2013). La universidad comunitaria intercultural de las nacionalidades y pueblos indí- genas "Amawtay Wasi" hacia un nuevo paradigma de educación superior en Educación intercultural a nivel superior. México: UIEP, UCIRED, UPEL.

Tünnermann, B.C. (2010). Universidad y sociedad: balance histórico y perspectivas desde Latinoamérica. Caracas: Universidad Central de Venezuela.

UNIBOL Guaraní y Pueblos de Tierras Bajas “Apiaguaiki Tüpa” (2009). Documento de organización curricular. Ministerio de Educación del Estado Plurinacional de Bolivia. La Paz-Bolivia: Área Académica UNIBOL.

Wachtel, N. (1973). Rebeliones y milenarismo. En: Juan M.Ossio (Ed.), Ideología mesiánica del mundo andino (118-123). Lima: Edición de Ignacio Prado Pastor.

Walsh, C. (2002). (De) construir la interculturalidad. Consideraciones críticas desde la política, la colonialidad y los movimientos indígenas y negros en el Ecuador. En: Norma Fuller (Ed.), Interculturalidad y política. Lima: Red de Apoyo de las Ciencias Sociales.

Weinberg, G. (1995). “Educación Prehispánica”. En su Modelos educativos en la historia de América latina. Buenos Aires: UNESCO/ CEPAL/PNUD.

Zambrana, A. (Ed.) (2014). Pluralismo epistemológico. Reflexiones sobre la educación superior en el Estado Plurinacional de Bolivia. Cochabamba: FUNPROEIB Andes - SAIH. 